\section{Comparison of the 2-D Dose Distribution Calculated by Planning System and Measured by Gafchromic Film Physical Dosimetry for ${ }^{60} \mathrm{Co}$ and ${ }^{192} \mathrm{Ir}$ Brachytherapy Sources}

\author{
Gholami M. H. ${ }^{1 \odot}$, Sadeghi M..3*๑, Babapour Mofrad F.4, \\ Mohammadi M.5,6
}

\begin{abstract}
Background: High Dose Rate (HDR) brachytherapy sources with high photon energy have been widely used in treating tumors. Dosimetric parameter of these brachytherapy sources should be determined according to the AAPM TG-43 recommendation. Gafchoromic films are reliable tools for this evaluation.
\end{abstract}

Objective: The aim of this study is to evaluate and compare dose accuracy of the two-brachytherapy sources in a dedicated phantom.

Material and Methods: In this analytical study, two common sources, including Cobalt and Iridium, were loaded into the dedicated phantom. The two-dimensional dose distribution around the source was calculated by TPS system for certain activities and geometries around the sources. Then, the experimental dose measured by Gafchromic film dosimetry was reported for different angles ranging from 0 to 180 degrees.

Results: The difference between calculated and measured doses was less than 6 percent $(-5$ to +6 percent) for all of the channels and angles. These errors are smaller and mainly more than zero $\left(D_{\text {film }}>D_{\text {TPS }}\right)$ for angles less than 20 and larger than 110 degrees. There is no statistically significant discrepancy in dose calculation by treatment planning system.

Conclusion: Although the estimated error in dose calculation is not significant, there is still an opportunity to increase the treatment precision. The correlation between the error and the angle should be considered in further plans of brachytherapy. The present study showed comparable errors compared to results of other research studies.

Citation: Gholami MH, Sadeghi M, Babapour Mofrad F, Mohammadi M. Comparison of the 2-D Dose Distribution Calculated by Planning System and Measured by Gafchromic Film Physical Dosimetry for ${ }^{60} \mathrm{Co}$ and ${ }^{192}$ Ir Brachytherapy Sources. J Biomed Phys Eng. 2020;10(3):299306. doi: 10.31661/jbpe.v0i0.1912-1004.

Keywords

Brachytherapy; Film Dosimetry; Cobalt-60; Iridium-192

\section{Introduction}

Preatment of cancer with ionizing radiation is a very common method in the medical practice [1]. The main goal in Radiation

L Therapy (RT) is to reach more treatment gains including the ratio of the dose received by the cancer tissue to that received by the healthy
${ }^{1} \mathrm{MSc}$, Department

of Medical Radiation

Engineering, Science

and Research Branch,

Islamic Azad University,

Tehran, Iran

${ }^{2} \mathrm{PhD}$, Finetech in Medi-

cine Research Center.

Iran University of Medi-

cal Sciences, Tehran,

Iran P.O. Box: 14155-

6183, Tehran, Iran

${ }^{3} \mathrm{PhD}$, Medical Physics

Department, School of

Medicine, Iran University

of Medical Sciences,

P.O. Box: 14155-6183,

Tehran, Iran

${ }^{4} \mathrm{PhD}$, Department

of Medical Radiation

Engineering, Science

and Research Branch,

Islamic Azad University,

Tehran, Iran

${ }^{5} \mathrm{PhD}$, Department of

Medical Physics, Royal

Adelaide Hospital, Ade-

laide, SA 5000 Australia

${ }^{6} \mathrm{PhD}$, School of Physical

Sciences, the University

of Adelaide, SA 5005

Australia

*Corresponding author:

M. Sadeghi

Finetech in Medicine

Research Center, Iran

University of Medical

Sciences, Tehran, Iran

P.O. Box: 14155-6183,

Tehran, Iran

E-mail: mahdisade-

ghi2003@yahoo.com

Received: 16 December 2019

Accepted: 8 January 2020 
tissue [2]. External beam RT (EBRT) usually imposes a considerable amount of radiation to healthy organs increasing the complications after RT in the organ at risks (OAR) along with the risk of secondary cancers [3]. In addition to the new approaches in EBRT like intensity modulated RT [4], there is a good and easily available opportunity in delivering the dose more wisely and precisely to the cancer tissue by means of brachytherapy [5], especially for prostate, cervix, and vaginal cases [6]. Due to the low time duration of treatments, High Dose Rate (HDR) brachytherapy with dose rates more than 12 Gy per hour has become more common recently for treating patients [7]. The source structure with dimensions of about a few centimeters made HDR brachytherapy more interesting to be used in clinics. The cobalt and iridium sources are being used in clinical practices with gamma energies of 1.38 and $0.8 \mathrm{MeV}$, respectively [8]. In treatment planning systems, choosing the source type and source activity, are critical factors affecting the dose distribution, the treatment response, and the post-RT complications [9]. On the other hand, it is critical to calculate the exact radiation dose imposed to healthy organs as well as tumors. These exact measurements and calculations leads to increasing the cancer dose to maximum extent without exceeding the tolerable doses related to important Organ at Risks (OARs). There are software and guidelines available to calculate the absorbed dose of any desired place in or adjacent to the treatment region. It is recommended to calibrate them and validate the output of these software to have better treatment responses and less complications in the clinic [10]. Physical measurement of the radiation dose prevents technical and calculation errors as well as post-RT problems [11]. Gafchromic film dosimetry is accepted in the dose verification $[12,13]$, especially in $2 \mathrm{D}$ dose distribution $[14,15]$. In this study, the aim is to compare the dose distribution and the dosimetric data of our treatment planning system (HDR Plus from Flexitron company) based on the American Association of Physicists in Medicine (AAPM) Task-Group 43 (TG 43) algorithm along with measurements of $2 \mathrm{D}$ radiation dose using Gafchromic film dosimetry for two commercially available sources of Cobalt-60 and Iridium-192.

\section{Material and Methods}

\section{Phantom Design}

For this analytical study, a phantom was designed and made to simulate the BALTAS phantom from Pi Medical Ltd Company. This phantom was made from plexiglass with the density of $1.2 \mathrm{gr} / \mathrm{cm}^{2}$. As shown in Figure 1,

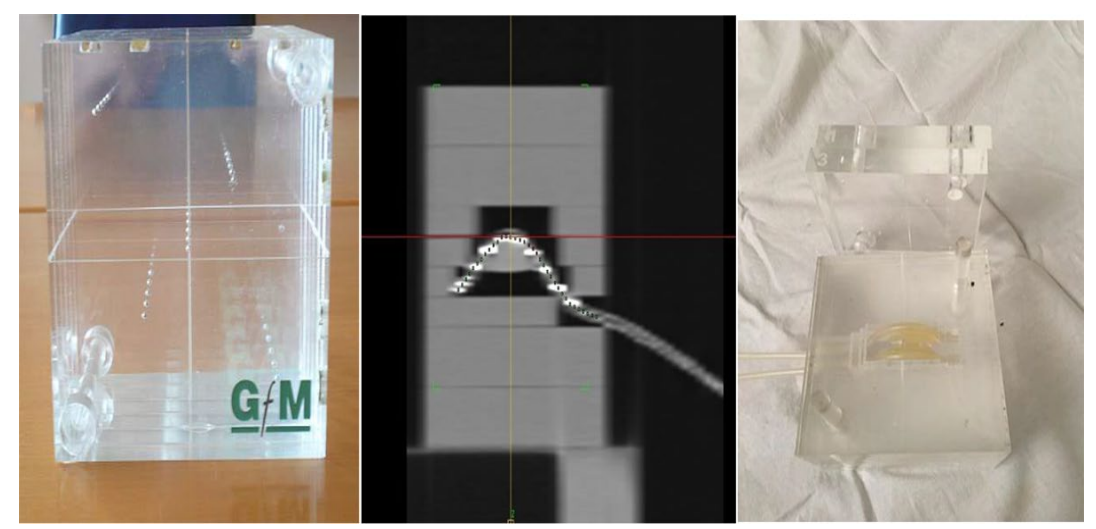

Figure 1: Dedicated phantom made in our lab and the applicator placed in it. 
there are 6 slabs of plexiglass and the thickness of each slab is $2 \mathrm{~cm}$. There are 4 rows of shots and one of them is in the central axis. The first (upper) layer consists of no shot. The $1^{\text {st }}$ and $2^{\text {nd }}$ rows of shots are $3 \mathrm{~cm}$ away from the central axis while the $3^{\text {rd }}$ and $4^{\text {th }}$ rows are $5.5 \mathrm{~cm}$ away.

The BALTAS phantom was modified to be suitable for the applicator and sources .This phantom contains several layers to seed placements. The source with the activity of $1.88 \mathrm{Ci}$ for Cobalt-60 and $6 \mathrm{Ci}$ for Iridium-192 was placed in determined points. Small rectangles with the sizes ranging from 2.5 to $4 \mathrm{~cm}^{2}$ were created using the laser incision in the $3^{\text {rd }}$ and $4^{\text {th }}$ slabs of phantom to place the applicator. The nasopharynx applicators were used to plant the seeds.

\section{Dose Calculation Formalism}

According to the recommendation of TG-43, the rate distribution of absorbed dose around a brachytherapy source can be determined using the following formula [16-19]:

$$
\dot{D}(r, \theta)=s_{k} \cdot \Lambda \cdot \frac{G(r, \theta)}{G\left(r_{0}, \theta_{0}\right)} \cdot g(r) \cdot F(r, \theta)
$$

Where $\boldsymbol{\Lambda}$ is the dose rate constant, $G(r, \theta)$ is the geometry function, $g_{L}(r)$ is the radial dose function, and $F(r, \theta)$ is the anisotropiy function.

The dose rate constant is obtained from equation 2 as follows:

$$
\Lambda=\frac{\dot{D}(r, \theta)}{s_{k}}
$$

The radial dose function, $g(r)$, describes attenuation in the tissue of photons emitted from source. The radial dose function is described as follows:

$$
g(r)=\frac{\dot{D}\left(r, \theta_{0}\right)}{\dot{D}\left(r_{0}, \theta_{0}\right)} \cdot \frac{G\left(r_{0}, \theta_{0}\right)}{G\left(r, \theta_{0}\right)}
$$

The geometry function also is defined as the following formula:

$$
G_{p}(r, \theta)=r^{-2}
$$

$$
G_{l}(r, \theta)=\left\{\begin{array}{ll}
\frac{\beta}{L r S i n \theta} & \text { if } \theta \neq 0 \text { degree } \\
\left(r^{2}-\frac{l^{2}}{4}\right)^{-1} & \text { if } \theta=0 \text { degree }
\end{array}\right\}
$$

" $\mathrm{L}$ " is the line source approximation used for the geometry function. $\beta$ is the angle, in radians, subtended by the tips of the hypothetical line source with respect to the calculation point $P(r, \theta)[20]$.

The anisotropy function $F(r, \theta)$, is defined as:

$$
F(r, \theta)=\frac{\dot{D}(r, \theta)}{\dot{D}\left(r, \theta_{0}\right)} \cdot \frac{G\left(r, \theta_{0}\right)}{G(r, \theta)}
$$

\section{Source description}

Applied HDR sources of the present study, Flexisource ${ }^{60} \mathrm{Co}$ and mHDR-v2 ${ }^{192} \mathrm{Ir}$ (Elekta, Netherlands), are shown in Figure 2. Moreover, the detailed information about the design and material components of both sources can be found in AAPM-ESTRO Report No. 229 [21].

Then, computed tomography (CT) images with $110 \mathrm{kVp}, 300 \mathrm{mAs}$, and $0.6 \mathrm{~mm}$ slice thickness were acquired to yield 3D images of X-Ray markers placed in the holes to measure and verify the accurate position and distance of the holes. The shots are made of metal material; therefore, they can be localized precisely on CT images. The 3 plane images were reviewed and the size and distances were measured carefully on Siemens CT workstation. The images were transported to treatment planning software HDR Plus (Flexiplan) to verify the software localization tool. The angular dose distribution in 5 layers were calculated using this software based on the TG43 AAPM report. The treatment plan then transported to the applicator using the Multi-score software. These calculations were recorded to 


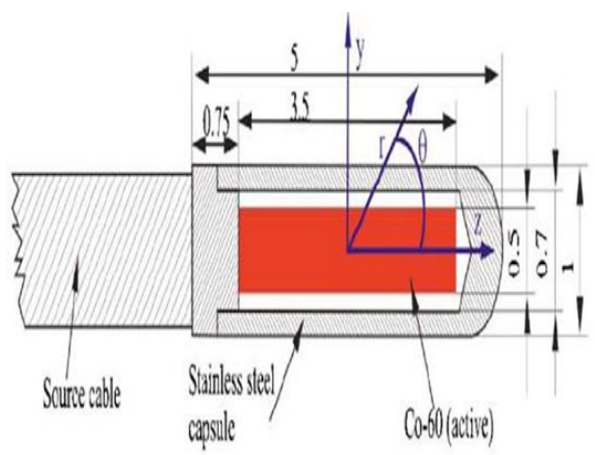

(a)

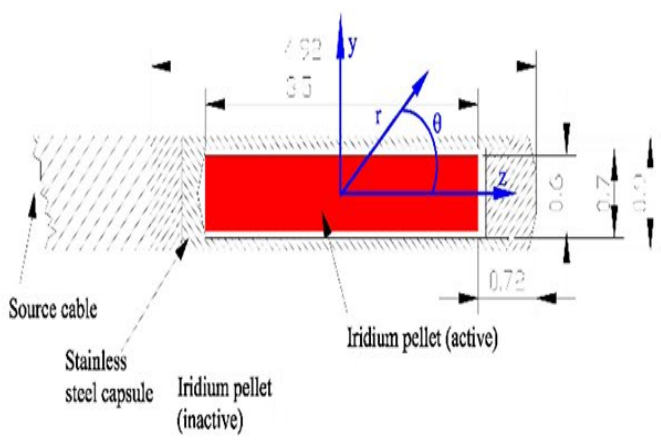

(b)

Figure 2: HDR sources of present study (a) Flexisource ${ }^{60} \mathrm{Co}$ and (b) mHDR-v2 ${ }^{192} \mathrm{Ir}$.

be compared with the film dosimetry measurements.

The standard calibration of Gafchromic was done by Shinva (6-MV) LINAC by exposing the film to doses ranging from 0.5 to $12 \mathrm{~Gy}$.

The two-dimensional dose distribution was assessed by measuring radial and angular functions along with film dosimetry. The Gafchromic EBT3 films prepared from Ashland company were used to measure the dose. The films were placed along the phantom as shown in Figure 3.

In 45 channels of angular distribution, the radiation dose was calculated and measured to assess the accuracy of TPS system in several positions.

Two sources of ${ }^{60}$ cobalt and ${ }^{192}$ Iridium were used to irradiate and compare with the activities of $1.88 \mathrm{Ci}$ and $6 \mathrm{Ci}$, respectively. The sources were situated in a dedicated place between $2^{\text {nd }}$ and $3^{\text {rd }}$ slabs and 10 seconds irradiation was implemented for both types of

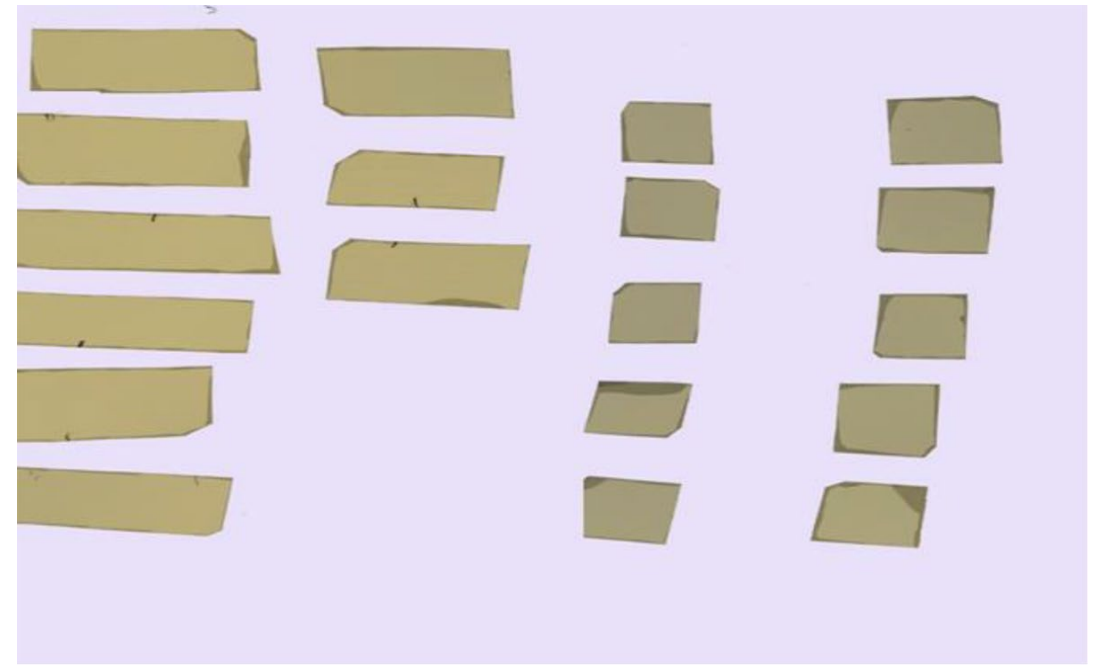

Figure 3: Calibration films 
sources.

\section{Statistical Analysis}

The difference between the calculated dose $\left(\mathrm{D}_{\text {TPS }}\right)$ and the measured dose $\left(\mathrm{D}_{\text {film }}\right)$ was investigated for all 45 channels of the angular distribution. The percent of relative error was defined as formula 1 . The errors were plotted and the paired T-test was used to compare them statistically. The Microsoft excel and SPSS IBM software were used to perform these calculations.

$$
R E=\frac{D_{\text {film }}-D_{T P S}}{D_{T P S}} \times 100(\text { percent })
$$

\section{Results}

There is negligible difference between two methods to estimate the $\mathrm{D}_{\text {TPS }}$ and $\mathrm{D}_{\text {film. }}$. The range of difference (in percent) was -5 percent to +6 percent error. There are angle-related changes in dose estimation errors which is not statistically significant (Paired test, $\mathrm{P}>0.05$ ). Figure 4 shows the RE of two sources used in this study. The horizontal axis shows the angle of measurement and the vertical axis is the relative error. The bias and the relative error in calculation are positive $\left(\mathrm{D}_{\text {film }}>\mathrm{D}_{\text {TPS }}\right)$ for small and large angles ( $<20$ and $>110)$, while the RE is lower than zero $\left(\mathrm{D}_{\text {film }}<\mathrm{D}_{\mathrm{TPS}}\right)$ for angles close to 50 degrees. The mean error and range of them for 5 channels are presented in Table 1, the mean error is the same for 5 channels and there is no statistically significant difference among them $(\mathrm{P}>0.05)$. There is no difference between RE between two sources of cobalt and iridium (student T-test. $\mathrm{P}>0.05$ ).

\section{Discussion}

Discrepancies between the calculated dose and the real dose were measured by simulating the situation using dedicated phantom. Other than the assessment of the average value, we did this experiment for a range of angular distribution to have reliable data using 2D film dosimetry. According to the angular distribution, there is a correlation between the angle and the relative error. This lack of accuracy

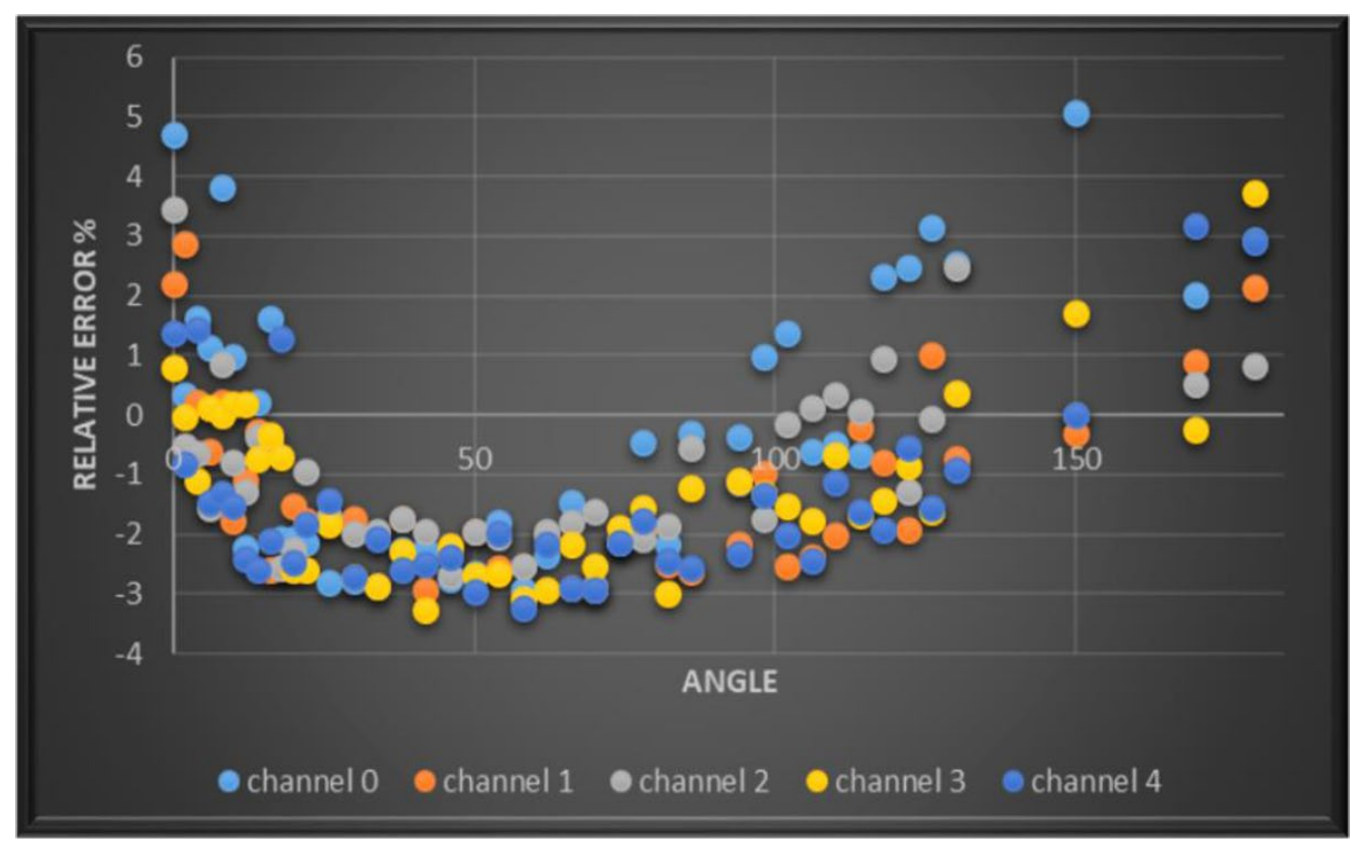

Figure 4: Average relative error percent for both of sources. 
Table 1: Error range and average for both of sources of Ir and Co.

\begin{tabular}{cccccc} 
& Channel 0 & Channel 1 & Channel 2 & Channel 3 & Channel 4 \\
\hline Absolute mean & 3.85 & 4.1 & 4.38 & 4.48 & 4.61 \\
\hline Max & 5.89 & 5.26 & 5.48 & 6.01 & 5.84 \\
\hline Min & 0.45 & 1.24 & 0.96 & 1.32 & 0.56
\end{tabular}

in dose calculation in certain angles can affect the treatment response, especially for the margins close to sensitive vulnerable healthy organs where the tolerance dose is very important. It should be considered according to the position of the calculation point, to ensure that the whole tumor receives adequate lethal dose while the healthy tissue damage remains at an acceptable level. There are uncertainties in the dose calculation conducted by TPS software. Some of the errors are predictable and some of them are random while the predictable bias can be corrected. The absolute error in the dose calculation compared with measurements is less than 6 percent, which is acceptable and consistent with former studies done using Gafchromic 2D dosimetry [17, 22-24]. Ayyobian et al. reported that the uncertainties in the dose calculation for Cobalt sources are lower than 4 percent [22] and Williamson reported 5 percent difference in calculation and measurements [17]. In the present study, a phantom suitable for the brachytherapy measurement and simulation was designed and made. The verification by 3D CT images shows accuracy and good details of the phantom. The calibration data were acceptable and curves were created to obtain the accurate dose data. The difference between our results and other same published data is lower than 2 percent. It can be concluded with confidence that clinical treatment in this study is in good agreement with other research studies. However, there are many sources of discrepancies. The errors can be due to errors in film calibration, TPS calculations, film maintenance, and calibra- tions in the source activity. This deviation may partly be attributed to the physical difference in the construction of the source models. In the cases with significant non-negligible RE, the treatment plan can be altered to obtain the accurate and reliable data of the dose distribution. Moreover, these data can be considered as calibration factors in TPS data or given to medical physicists and radiation oncologists as a guideline to avoid discrepancies.

\section{Conclusion}

The dose calculations carried out based on treatment planning systems using two most common sources of 60-Cobalt and 192-Iridium in the clinical practice are reliable in treatments of patients and the accuracy of treatment and calculation can increase by calculating calibration factors along with the ratio of measurement and TPS output for different angles.

\section{Conflict of Interest}

None

\section{References}

1. Chen HH, Kuo MT. Improving radiotherapy in cancer treatment: promises and challenges. Oncotarget. 2017;8(37):62742. doi: 10.18632/ oncotarget.18409. PubMed PMID: 28977985. PubMed PMCID: PMC5617545.

2. Vuong T, Devic S, Moftah B, Evans M, Podgorsak EB. High-dose-rate endorectal brachytherapy in the treatment of locally advanced rectal carcinoma: technical aspects. Brachytherapy. 2005;4(3):230-5. doi: 10.1016/j. brachy.2005.03.006.

3. Devic S, Liang L, Tomic N, Bekerat H, Morcos 
2-D Dose Distribution of ${ }^{60} \mathrm{Co}$ and ${ }^{192} \mathrm{Ir}$

M, Popovic M, Watson P, Aldelaijan S, Seuntjens J. Dose measurements nearby low energy electronic brachytherapy sources using radiochromic film. Physica Medica. 2019;64:40-4. doi: 10.1016/j.ejmp.2019.05.017.

4. Van Der Veen J, Laenen A, Nuyts S. Modern radiotherapy techniques versus three-dimensional conformal radiotherapy for head and neck cancer. Cochrane Database of Systematic Reviews. 2017;(12):1-12. doi: 10.1002/14651858. CD012904.

5. DeLaney TF, Chen GT, Mauceri TC, Munro III JJ, Hornicek FJ, Pedlow FX, Suit HD. Intraoperative dural irradiation by customized 192iridium and 90yttrium brachytherapy plaques. International Journal of Radiation Oncology Biology Physics. 2003;57(1):239-45. doi: 10.1016/S03603016(03)00505-4.

6. Martin E, Sowards K, Wang B. Investigation of a source model for a new electronic brachytherapy tandem by film measurement. Journal of applied clinical medical physics. 2018;19(5):640-50. doi: 10.1002/acm2.12440.

7. Wilkinson DA. High dose rate (HDR) brachytherapy quality assurance: a practical guide. Biomed Imaging Interv J. 2006;2(2):e34. doi: 10.2349/ biij.2.2.e34.

8. Meisberger LL, Keller RJ, Shalek RJ. The Effective Attenuation in Water of the Gamma Rays of Gold 198, Iridium 192, Cesium 137, Radium 226, and Cobalt 60. Radiology. 1968;90(5); 953-7. doi: 10.1148/90.5.953. PMID: 5643598.

9. Leung TW, Tung SY, Sze WK, Sze WM, Wong VY, Sai-Ki O. Salvage brachytherapy for patients with locally persistent nasopharyngeal carcinoma. International Journal of Radiation Oncology Biology Physics. 2000;47(2):405-12. doi: 10.1016/S0360-3016(00)00463-6.

10. Nath R, Anderson LL, Meli JA, Olch AJ, Stitt JA, Williamson JF. Code of practice for brachytherapy physics: report of the AAPM Radiation Therapy Committee Task Group No.56. Medical physics. 1997;24(10):1557-98.

11.Zaman ZK, Ung NM, Malik RA, Ho GF, Phua VC, Jamalludin Z, Baharuldin MT, Ng KH. Comparison of planned and measured rectal dose in-vivo during high dose rate Cobalt-60 brachytherapy of cervical cancer. Physica Medica. 2014;30(8):9804. doi: 10.1016/j.ejmp.2014.07.002.

12. Kirisits C, Rivard MJ, Baltas D, Ballester F, De Brabandere $M$, Van Der Laarse $R$, Niatsetski $Y$,
Papagiannis P, Hellebust TP, Perez-Calatayud J, Tanderup K. Review of clinical brachytherapy uncertainties: analysis guidelines of GEC-ESTRO and the AAPM. Radiotherapy and oncology. 2014;110(1):199-212. doi: 10.1016/j.radonc.2013.11.002.

13. Luvizotto J. Characteristics of the radiochromic film Gafchromictm EBT3 model for use in brachytherapy. Brazil: INIS; 2015;47(10):1-103.

14. Devic $S$. Radiochromic film dosimetry: past, present, and future. Physica medica. 2011;27(3):12234. doi: 10.1016/j.ejmp.2010.10.001.

15. Devic S, Tomic N, Lewis D. Reference radiochromic film dosimetry: review of technical aspects. Physica Medica. 2016;32(4):541-56. doi: 10.1016/j.ejmp.2016.02.008.

16. Meigooni AS, Gearheart DM, Sowards K. Experimental determination of dosimetric characteristics of Best $\AA$ brachytherapy source. Medical physics. 2000;27(9):2168-73. doi: 10.1118/1.1289256.

17. Williamson JF. Comparison of measured and calculated dose rates in water near $\mathrm{I}-125$ and Ir192 seeds. Medical physics. 1991;18(4):776-86. doi: 10.1118/1.596631.

18. Wallace RE, Fan JJ. Report on the dosimetry of a new design brachytherapy source. Medical physics. 1999;26(9):1925-31. doi: 10.1118/1.598737.

19. Meigooni AS, Yoe-Sein MM, Al-Otoom AY, Sowards KT. Determination of the dosimetric characteristics of InterSource125 iodine brachytherapy source. Applied Radiation and Isotopes. 2002;56(4):589-99. doi: 10.1016/S09698043(01)00258-5.

20. Rivard MJ, Coursey BM, DeWerd LA, Hanson WF, Saiful Huq M, Ibbott GS, et al. Update of AAPM Task Group No. 43 Report: A revised AAPM protocol for brachytherapy dose calculations. Medical physics. 2004;31(3):633-74. doi: 10.1118/1.1646040.

21. Perez-Calatayud J, Ballester F, Das RK, DeWerd LA, Ibbott GS, Meigooni AS, et al. Dose calculation for photon-emitting brachytherapy sources with average energy higher than $50 \mathrm{keV}$ : report of the AAPM and ESTRO. Medical physics. 2012;39(5):2904-29. doi: 10.1118/1.3703892.

22. Ayoobian N, Asl AS, Poorbaygi $H$, Javanshir MR. Gafchromic film dosimetry of a new HDR brachytherapy source. Journal of applied clinical medical physics. 2016;17(2):194-205. doi: 
Gholami M. H. et al

10.1120/jacmp.v17i2.6005.

23. Bastin KT, Podgorsak MB, Thomadsen BR. The transit dose component of high dose rate brachytherapy: direct measurements and clinical implications. International Journal of Radiation Oncology Biology Physics. 1993;26(4):695-702. doi: 10.1016/0360-3016(93)90291-3.

24. Richter J, Baier K, Flentje M. Comparison of 60 Cobalt and 192 Iridium sources in high dose rate afterloading brachytherapy. Strahlentherapie und Onkologie. 2008;184(4):187-92. doi: 10.1007/s00066-008-1684-y. 